\title{
Ultraviolet All-Optical Switching via Zinc Oxide Thin Films
}

\author{
Trenten A. Smith ${ }^{1}$, Samuel J. Haeuser ${ }^{2}$, Seth T. King ${ }^{3}$, and Eric J. Gansen ${ }^{4}$ \\ ${ }^{1,2}$ Student (Physics Department, University of \\ Wisconsin-La Crosse) \\ La Crosse, WI, USA \\ ${ }^{3,4}$ Professor (Physics Department, University of \\ Wisconsin-La Crosse) \\ La Crosse, WI, USA
}

\begin{abstract}
Zinc oxide $(\mathrm{ZnO})$ is a semiconductor material exhibiting a wide bandgap in the ultraviolet (UV) region. $\mathrm{ZnO}$ is a promising material for use in short-wave optoelectronic devices such as all-optical switches (AOSs). Our switch is composed of a polycrystalline $\mathrm{ZnO}$ thin film grown by DC sputter deposition and uses a 120ps control pulse tuned to the band edge of the film to modify the transmission of a weaker signal pulse. The signal light is heavily absorbed in the absence of the control pulse, representing an off state of the switch. The control pulse, when incident on the film, resonantly excites electron-hole pairs. This decreases the material's absorption by filling energy states and screening the built-in electric field of the $\mathrm{ZnO}$. Consequently, more signal light is transmitted by the film, representing an on state.
\end{abstract}

\section{Introduction}

Today, there is a growing interest in the advancement of free-space communications, displays, and data storage through the development of optoelectronic devices operating in the ultraviolet (UV) region. These developments are integral to NASA's directorates in human exploration, aeronautics research, and basic science (NASA, 2014). Zinc oxide $(\mathrm{ZnO})$ is a semiconductor material exhibiting a wide bandgap in the UV spectral region which makes it a promising material for shortwave optoelectronic devices such as an all-optical switch (AOS).

Switches are fundamental components to communication systems and are used to encode information via some medium. In an optical communication system, this medium is a beam of light or a train of light pulses. Our switch consists of a $\mathrm{ZnO}$ material system that modulates the amount of light passed through the device; this determines the on and off states. $\mathrm{ZnO}$ exhibits a built-in field and an exciton resonance occurring near $376 \mathrm{~nm}$ making it an ideal optical switch in the UV region. Our AOS utilizes the Stark Effect where the exciton absorption resonances of the $\mathrm{ZnO}$ are shifted to lower energies by a strong control pulse. Short-wave devices, like those constructed from $\mathrm{ZnO}$ structures, are particularly well suited for high density (HD) data storage (e.g. Blu-ray discs) and non-line-of-sight (NLOS) UV communication systems.

Previous investigations of control-signal transmission dynamics of $\mathrm{ZnO}$ have focused largely on structures grown by plasma-assisted molecular beam epitaxy (MBE). While this growth method results in single-crystal films, it is not suitable for large-scale commercial production. We have been developing short-wave AOSs through the study of the optoelectrical properties of $\mathrm{ZnO}$ films grown by DC sputter deposition. By contrast to MBE, sputter deposition can be directly scaled to industry-level production. The absorption of our device is the key factor in creating an AOS. The 
properties of the $\mathrm{ZnO}$ thin-film structures have been investigated via structural composition and temperature dependence, and thus have led us to an AOS that utilizes absorption.

\section{Background}

2.1 Semiconductor Basics and the Stark Effect. A semiconductor is a type of material with a bandgap large enough that the electrons cannot freely flow from the valence band (VB) into the conduction band (CB), but small enough where the electrons can still be excited into the conduction band. A photon with energy greater than the bandgap energy ( $\left.E_{g a p}\right)$ of a semiconductor can be absorbed, thus exciting an electron from the valence band to the conduction band, as shown in Figure 1(a). This new electron vacancy in the valence band is referred to as a hole. The absence of a negative charge acts similarly to the presence of a positive charge. A bound electron-hole pair is known as an exciton, shown in Figure 1(b). The Stark Effect is characterized by a shift in the band edge towards lower energies, which corresponds to higher wavelengths (red shift), in the presence of a static electric field, as illustrated in Figure 1(c). While the electric field must be applied for some semiconductors, $\mathrm{ZnO}$ has a built-in electric field. In our AOS, the Stark Effect is used to modulate the transmission of light pulses tuned near the band edge of the structure.

(a)

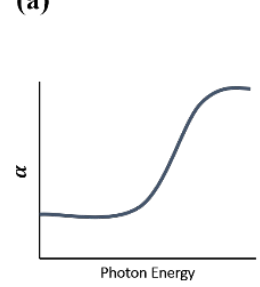

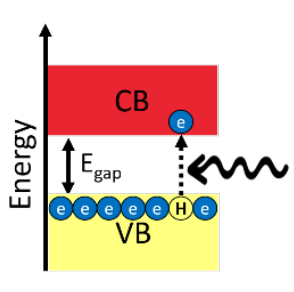

(b)

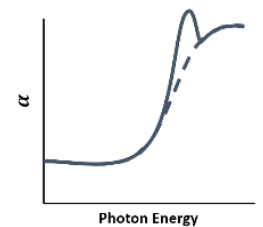

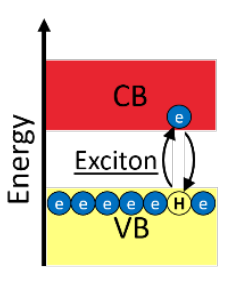

(c)
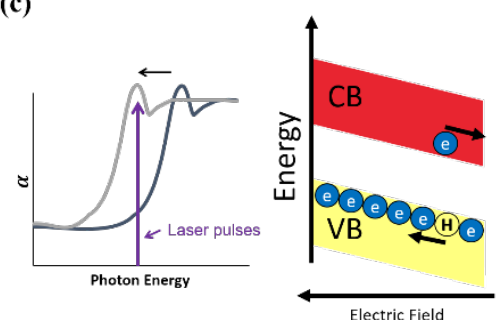

Figure 1. Dynamics involved with absorption $(\alpha)$ and the Stark Effect in semiconductors. (a) Photon with energy greater than the bandgap energy of a semiconductor is absorbed causing an electron to jump from the valence to the conduction band. (b) Exciton formation causing a resonance near the bandgap of the material. (c) Shifting of the band edge due to the presence of a static electric field.

2.2 All-optical switching. The main function of an AOS is to imprint information on a beam of light or sequences of light pulses, as illustrated in Figure 2(a). An AOS can be constructed by spatially overlapping the signal pulse with the control pulse, which is used to change the absorption properties of the material by exciting electrons. By utilizing a change in its absorption coefficient $(\alpha)$, our semiconductor AOS controls the state of the switch. The change in absorption causes a change in the amount of signal light transmitted by the switch producing well-defined on and off states lasting as long as the electrons remain excited in the material. Much like a hydrogen atom, the excitons of our AOS exhibit discrete energy levels (i.e. resonances) in their absorption spectra. Shown in Fig. 2(b) are these features which make $\mathrm{ZnO}$ thin films suitable semiconductor AOSs. By tuning the incident light near the exciton resonance, our AOS uses a Stark Effect. 

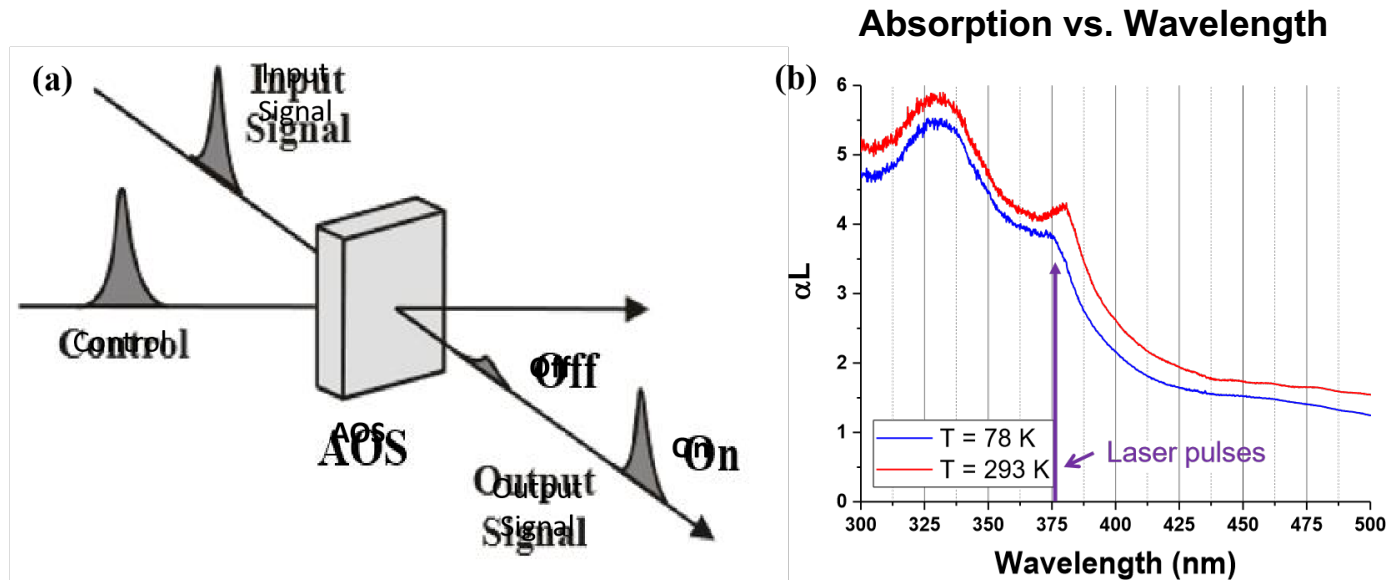

Figure 2. (a) Schematic diagram of an all-optical switch. The on/off state of the switch is determined by the presence of the control and the signal pulses. (b) Absorption spectra of our ZnO AOS showing our laser pulse tuned at $376 \mathrm{~nm}$ centered near the exciton resonance.

The Stark Effect is utilized in our AOS, as illustrated in Figure 3 (Miller et al., 1985). In this device, the $\mathrm{ZnO}$ thin film acts as a switching material exhibiting a built-in static electric field ( $\mathrm{E}_{\text {built }}$ ). The static field bends the conduction and valence bands, and through the Stark Effect, shifts the exciton resonances to lower energies (red shift). As indicated by the vertical arrows in Fig. 3, all-optical switching can be achieved by tuning the laser pulses to the redshifted exciton resonance and by using the control pulse to modify the absorption. Prior to the arrival of the control pulse, shown in Fig. 3(a), the resonantly tuned signal light is highly absorbed. Because the light is highly absorbed, very little light transmits through, constituting the off state of the switch. As shown in Fig. 3(b), the control pulse is absorbed and excites electrons to create electron-hole pairs in the thin film. This fills some of the available exciton states reducing the absorption experienced by the signal pulse due to Pauli blocking (Chemla, Miller, 1985). This initial "bleaching" of the exciton resonance lasts for as long as the states remain filled. Subsequently, the electrons and holes break apart, and are pulled in opposite directions due to the internal static electric field. As illustrated by Fig. 3(c), this charge separation produces a space-charge field $\left(E_{s c r}\right)$ that screens the static field, thus shifting the excitonic resonances to higher energies (blue shift). The decrease in absorption is typically greater due to the blue shift than by bleaching. The time it takes for the device to fully turn on, commonly referred to as the sweep-out time, depends on how fast the carriers break apart and travel through the sample. Sweep-out times of 1-100's of ps are typical (Livescu et al., 1989; Mahgerefteh et al., 1992; Yairi et al., 1999) in this type of optical switch.

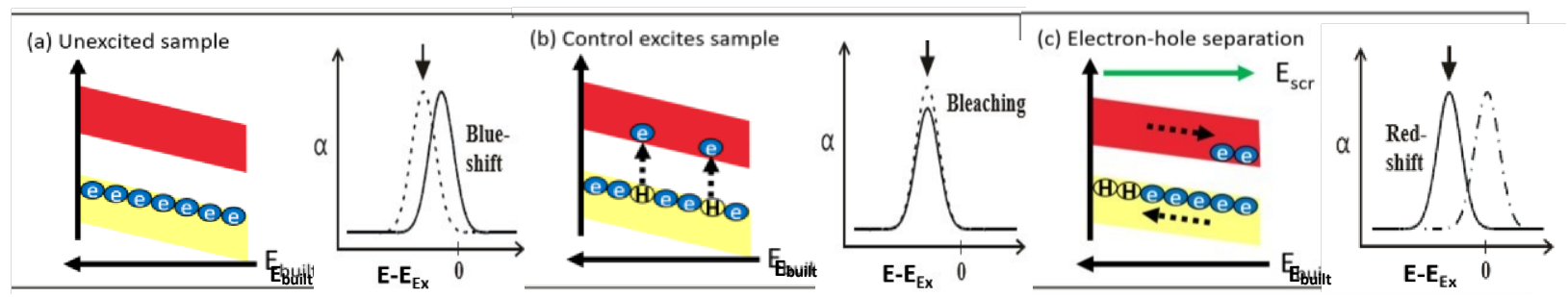

Figure 3. Principals of operation for AOSs based on the Stark Effect. (a) The unexcited $\mathrm{ZnO}$ thin film, showing the bent energy bands and the redshifted exciton resonance (solid line) caused by the built-in static electric field Ebuilt. The absorption resonance in the absence of Ebuilt is represented by the dash-dotted curve centered at EEx. (b) When incident, the control pulse excites electron-hole 
pairs in the thin film. This initially bleaches the exciton resonance (solid line). (c) The electrons and holes separate due to $E_{b u i l t}$ producing a space charge field ( $\left.E_{s c r}\right)$ that screens $E_{b u i l t}$, thus blue shifting the exciton resonance. The AOS is in the on state until the electron-hole pairs recombine or until they are swept out of the semiconductor. In (b-c) the absorption resonance prior to excitation is represented by the dashed curve. The vertical arrows over the absorption spectra indicate the tuning of the control and signal pulses.

The turn-off time of the device is the time it takes to return to an off state. This depends on the time it takes for the space-charge field to decay. For tightly focused control and signal pulses, turn-off times on the order of 10's of ps have been observed (Livescu et al., 1989; Mahgerefteh et al., 1992; Yairi et al., 1999) due to enhanced in-plane transport of the carriers (McCallum et al., 1993). Here, the electrons and holes responsible for the space-charge field disperse laterally on a time scale faster than that allowed by conventional carrier diffusion. Because electron-hole pairs are only created within the illuminated area of the MQW, the static field, $\mathrm{E}_{\text {built, }}$, responsible for bending the bands is only screened over a finite region of the structure. Consequently, potential gradients are formed, which drive the electrons and holes laterally out of the excitation region. By using a very tightly focused control beam, the potential gradients are very large, and the carrier distributions disperse very quickly. This results in a much faster turn-off time for the device than would be obtained by conventional in-plane diffusion or by recombination of the electrons and holes. For example, Yairi et al. (1999) reported a total switching time of $\sim 50 \mathrm{ps}$ for such a device.

2.3 The need for short-wave AOSs. The band-gap energy of materials chosen for AOSs determine the operating wavelength. For example, gallium arsenide/aluminum gallium arsenide (GaAs/AlGaAs) multiple quantum well (MQW) semiconductors exhibit a band-gap energy of $\sim 1.46 \mathrm{eV}$ at room temperature and are used to construct modulators operating in the near-infrared region $(\sim 850 \mathrm{~nm})$ (Goossen, 1994). Alternative materials are used to create modulators that operate in the infrared (IR) wavelengths used in fiber-optic communication systems. For example, devices operating at $1.3 \mu \mathrm{m}$ (O-band) have been constructed using germanium/silicon germanium (Ge/SiGe) MQWs (Kuo et al., 2005; Chaisakul et al., 2010; Lever et al., 2011; Chaisakul et al., 2012). These modulators are essential for developing fast and efficient optical interconnections between integrated circuits.

The enhanced spatial resolution possible with short-wavelength light $(350 \mathrm{~nm}-550 \mathrm{~nm})$ and the further development of UV NLOS communications drive the interest in the UV AOSs that we are developing. This research directly aligns with NASA's Space Technology directorates that are responsible for developing the crosscutting technologies and capabilities needed to achieve NASA's missions. Communicating in the UV region has proven to be advantageous through free space channels. There are low amounts of UV solar radiation within Earth's atmosphere leading to low background radiation for ground-based receivers. There is also an abundance of communication paths even if the receiver is not in sight of the transmitter. This is because there is a large scattering cross section of the molecules and aerosols with UV light. UV systems have the ability to transmit information at higher data rates than IR communication systems, and they are known to be more secure than their traditional radio frequency (RF) counterparts. This allows for UV NLOS communications to be well equipped for a number of civilian and military applications such as data communication, surveillance sensor networks, homeland security, unattended ground sensor (UGS) networks, and small unit communications in urban terrain environment (Xu el al., 2007). Short-wavelength modulators can be used to increase density for optical data storage as 
exemplified by "Blu-ray disc" technology. The integrated circuits industry uses short-wavelength light to write masks for integrated circuits with reduced feature size. Another application for $\mathrm{ZnO}$ AOSs is transparent displays. Optoelectronic devices used for transparent displays need to be transparent to most of the visible wavelengths; thus, they operate at shorter wavelengths near the UV spectral range.

Constructing modulators that operate in the green to UV spectral range requires a material system with a much wider bandgap than GaAs or Ge. Current research on optoelectronic devices within this spectral window has used nitride-based materials such as gallium nitride $(\mathrm{GaN})$. With a wide bandgap $\left(\mathrm{E}_{\mathrm{g}} \sim 3.4 \mathrm{eV}\right)$, a large exciton binding energy $(\sim 28 \mathrm{meV})$, and the ability to be $p$ and $n$ doped to produce $\mathrm{p}-\mathrm{n}$ junction diodes used in electrically pumped blue/UV lasers, $\mathrm{GaN}$ has drawn great attention in recent research. An attractive, non-toxic alternative to $\mathrm{GaN}$ material systems are those based on $\mathrm{ZnO}$. Along with being a safer substance than $\mathrm{GaN}, \mathrm{ZnO}$ is much less expensive while sharing many of the same optoelectronic characteristics. Both materials are wide bandgap semiconductors $\left(\mathrm{E}_{\mathrm{g}} \sim 3.4 \mathrm{eV}\right)$, and $\mathrm{ZnO}$ has an even larger exciton binding energy $(\sim 60 \mathrm{meV})$. Zinc oxide can be directly integrated with GaN lasers and LEDs because of the almost perfect lattice matching (Zhang et al., 2007; Sato et al., 2012). Recent studies on $\mathrm{ZnO} / \mathrm{Zn}_{0.95} \mathrm{Mg}_{0.05} \mathrm{O} \mathrm{MQWs}$ grown by plasma-assisted MBE produced promising results (Sato et al., 2012). However, this method of growth cannot be scaled to commercial production like sputter deposition. Our AOS is a simpler thin film design grown by DC sputter deposition and has shown promising switching capabilities.

\section{Experimental Results}

A schematic of the control-signal experimentation is shown in Figure 4(a). The ultraviolet laser used is a $120 \mathrm{ps}$ pulsed photodiode centered at $376 \mathrm{~nm}$, and it has a $40 \mathrm{MHz}$ repetition rate. The beam is directed at a $93 / 7$ beam splitter (BS-1) where 93 percent of the pulse intensity is directed as the control pulse. The remaining 7 percent is reflected as the signal pulse. We use a strong control pulse to result in sizable state filling effects in the AOS. The control and signal are optically chopped at $30 \mathrm{~Hz}$ and $1000 \mathrm{~Hz}$, respectively. After a series of optics, the pulses interact with the $\mathrm{ZnO}$ thin film. Finally, the signal pulse is captured by a photo-multiplier tube (PMT) detector. The output of this detector is sent to a cascaded lock-in amplifier system where we can read transmission (T) data. The first amplifier is locked at $1000 \mathrm{~Hz}$ whose output is sent to the second amplifier locked at $30 \mathrm{~Hz}$. This allows us to view a pure signal on the first lock-in, and then determine the percent change in the signal with the second $(\Delta T / T)$ as well as reduce noise in the system.

A variable delay stage allows us to vary the path difference between the control and signal paths, and thus vary the time delay $(\tau)$ between the control and signal pulses. Depending on the position of the variable delay stage, there is spatial overlap of the pulses. Coincident control and signal pulses is regarded as a time delay of zero. A positive $\tau$ indicates the control pulse interacting with the AOS prior to the signal pulse. Figure 4(b) shows the structure of our AOS. Our active layer is a $120 \mathrm{~nm} \mathrm{ZnO}$ thin film surrounded by two buffer layers. The $\mathrm{ZnMgO}$ buffer is used to ease the growth process and to protect the $\mathrm{ZnO}$ thin film. The other layers are used to create a capacitor like system. This allows us to change the static electric field in the AOS. 

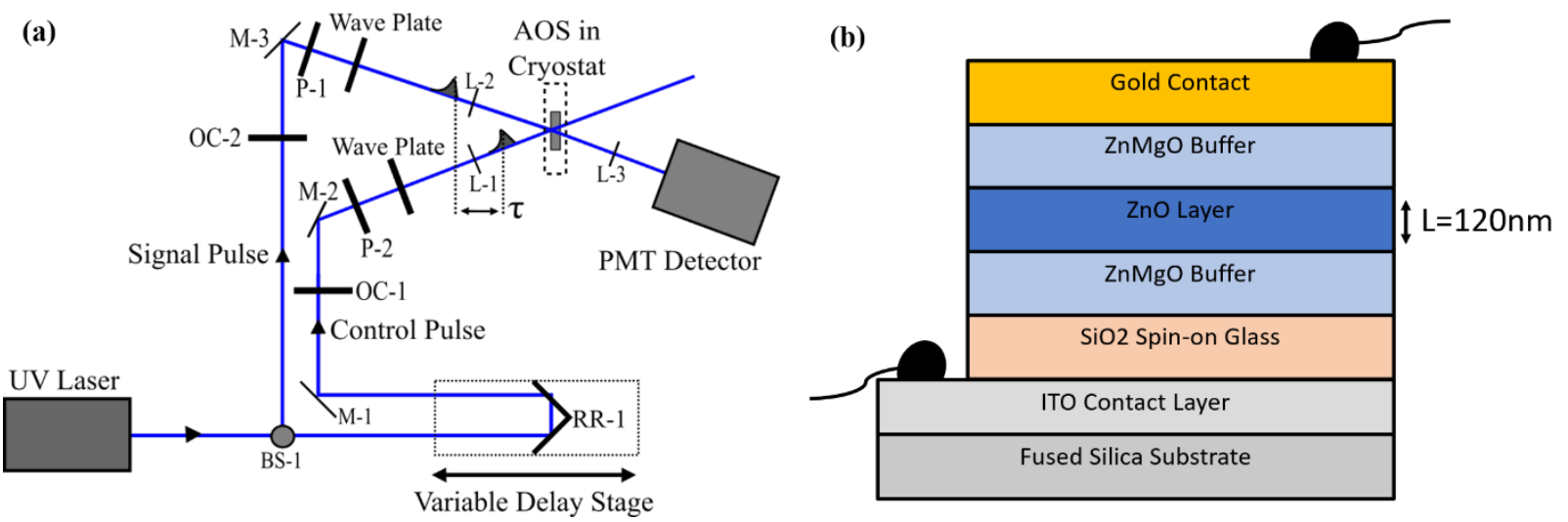

Figure 4. (a) Schematic of the control-signal experimental setup. The laser is a 120ps pulsed UV photodiode laser centered at $376 \mathrm{~nm}$ with a $40 \mathrm{MHz}$ repetition rate. The optics used include a beam splitter (BS), a retroreflector (RR), mirrors (M), optical choppers (OC), polarizers (P), lenses (L), and a photo-multiplier tube (PMT) detector. The time delay $(\tau)$ is determined by the variable delay stage. (b) Schematic of our $\mathrm{ZnO}$ AOS which has a $120 \mathrm{~nm} \mathrm{ZnO}$ active layer. This schematic is not to scale, and the other thicknesses are not relevant to the function of the AOS.

To assist in our data collection, we have developed a program on LabVIEW. This engineering software moves the delay stage and collects data from both lock-in amplifiers at specified increments. In Fig. 5, we plot $\Delta \mathrm{T} / \mathrm{T}$ as a function of $\tau$ with the AOS cooled to $78 \mathrm{~K}$ and kept at room temperature (273K). The control pulse and integrated control are overlaid on the graph to illustrate the accumulation of excited carriers. While we observe control-induced changes in the signal transmission for both temperatures, a much larger change is observed at $78 \mathrm{~K}$ than at room temperature. In addition, a more obvious time-delay dependence is observed at $78 \mathrm{~K}$ than at room temperature. We observe a maximum change in transmission at a slightly positive time delay that corresponds with the climax of the integrated control pulse when the number of excited carriers is expected to be optimized. The AOS does not fully recover within the $25 \mathrm{~ns}$ between pulses indicating that the built-in field is inhibiting recombination by spatially separating the electrons and holes. The fact that little time-delay dependence is observed at room temperature could be a consequence of the much smaller signal or could be a result of modified carrier dynamics at the elevated temperature. 


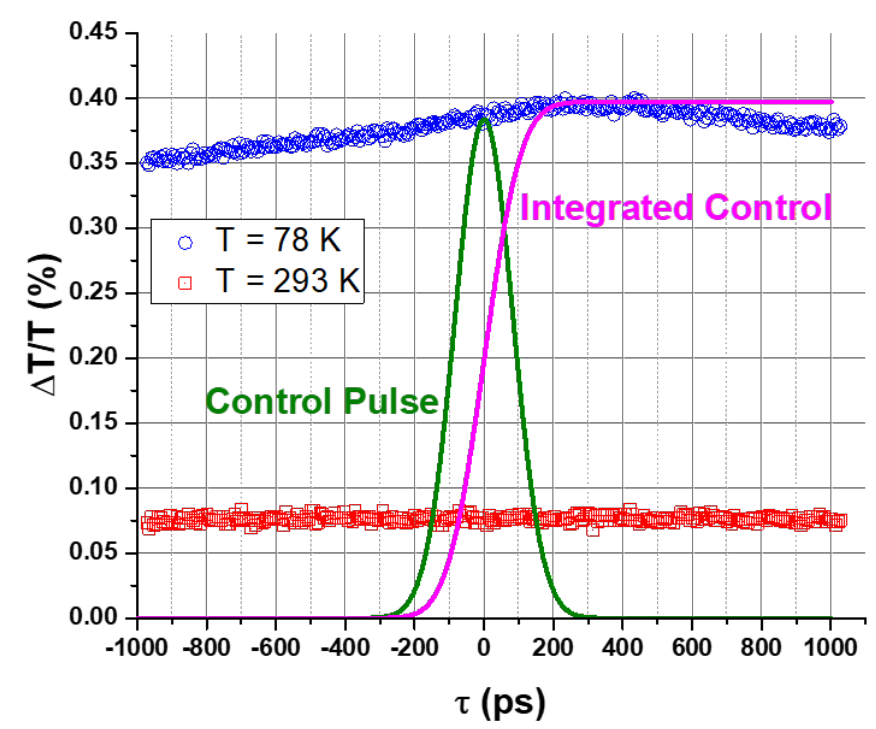

Figure 5. Graph of control-induced change in signal transmission measured as a percent of the change in transmission over the transmission of the signal $(\Delta \mathrm{T} / \mathrm{T})$ as a function of the time delay $(\tau)$. Positive delay indicates the control pulse interacting with the AOS prior to the signal pulse.

Evident by Fig. 6(a), the effectiveness of switching increases with an increasing control power. We also see a nonlinear relationship between the change of transmission and the control power. From Fig. 6(b), we see very little polarization $(\theta)$ dependence of the AOS. A graphic of our definition of $\theta$ is included on the graph for greater understanding.

(a)

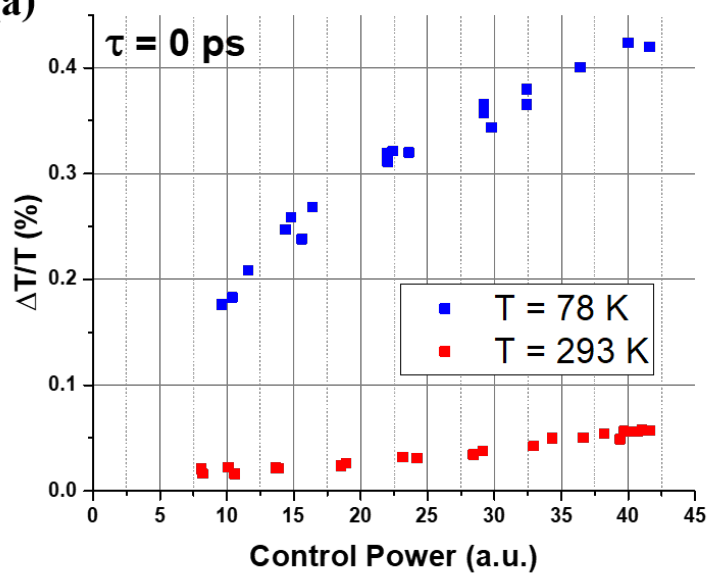

(b)

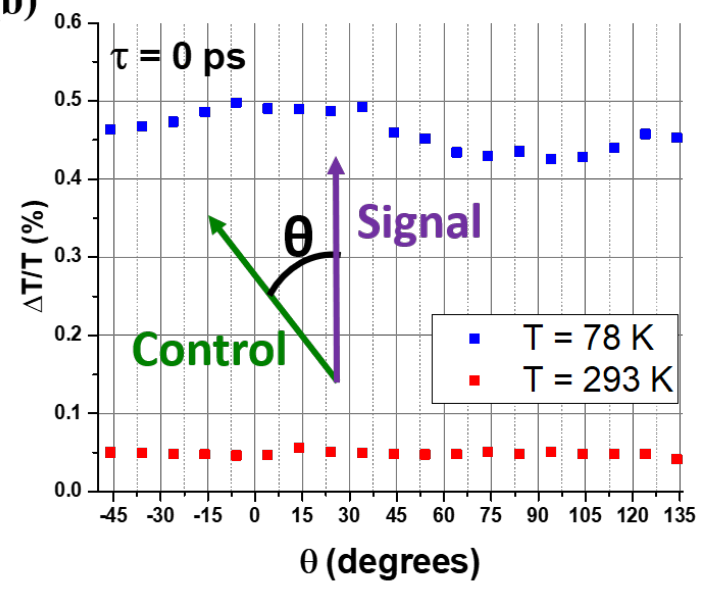

Figure 6. (a) Graph of $\Delta \mathrm{T} / \mathrm{T}$ versus the control power. A nonlinear relationship is observed at $78 \mathrm{~K}$. (b) Graph of $\Delta \mathrm{T} / \mathrm{T}$ versus the polarization $(\theta)$. A graphic of the definition of $\theta$ is included.

\section{Conclusion}

We have demonstrated all-optical switching using a $\mathrm{ZnO}$ thin film. Using a control-signal experimental setup, we demonstrated switching capabilities to create a change in transmission of greater than 0.4 percent. However, the AOS does not fully recover. Shown in Fig. 5, the delayed recovery of our switch is consistent with the charge separation induced by the built-in electric field. 
The increase in the change of transmission observed at lower temperatures is consistent with the relaxation of excited carriers to the bottom of the band and the sharpening of the absorption spectra as seen in Figure 2(b). A dependence of the switching on the density of the carrier population created by the control pulse is demonstrated through both time delay and power experiments. The increase in effectiveness of the switch with increasing power is due to the increase in excited electrons created by the control pulse. The nonlinear result indicates a saturation of exited electrons within the material. We also found very little dependence on the polarization of the control and signal. This is consistent with switching caused by state filling and screening effects as opposed to polarization switching. Our ZnO thin film grown by DC sputter deposition utilizes the Stark Effect and demonstrates promise as an all-optical amplitude switch. Future work will include optimizing the experimental system and software as well as testing different samples varying in thicknesses and composition.

\section{Acknowledgements}

Trenten Smith would like to acknowledge the Wisconsin Space Grant Consortium Undergraduate Research Award Program for their financial support. Samuel Haeuser would like to acknowledge the UW-La Crosse Dean's Distinguished Fellowship Program for their financial support. We would also like to acknowledge Dr. Eric Gansen for his mentoring, Dr. Seth King for growing our structures, and the University of Wisconsin-La Crosse for allowing us to use their facilities.

\section{References}

Chaisakul P, Marris-Morini D, Isella G, Chrastina D, Le Roux X, Gatti E, Edmond S, Osmond J, Cassan E, and Vivien L. (2010) "Quantum-confined Stark effect measurements in Ge/SiGe quantum-well structures," Opt. Lett. 35, 2913.

Chaisakul P, Marris-Morini D, Rouified MS, Isella G, Chrastina D, Frigerio J, Le Roux X, Edmond S, Coudevylle JR, and Vivien L. (2012) “23 GHz Ge/SiGe multiple quantum well electro-absorption modulator,” Opt. Exp. $20,3219$.

Chemla, DS, and Miller, DAB. (1985) "Room-temperature excitonic nonlinearoptical effects in semiconductor quantum-well structures." Journal of the Optical Society of America B 2, 1155.

Goossen KW Cunningham JE, and Jan WY. (1994) "Electroabsorption in ultranarrow-barrier GaAs/AlGaAs multiplequantum-well modulators," Appl. Phys. Lett., 64, 1071.

Kuo YH, Lee YK, Ge Y, Ren S, Roth JE, Kamins TI, Miller DAB, and Harris JS. (2005) "Strong quantum-confined Stark effect in germanium quantum-well structures on silicon," Nature 437, 1334.

Lever L, Hu Y, Myronov M, Liu X, Owens N, Gardes FY, Marko IP, Sweeney SJ, Ikonic Z, Leadley DR, Reed GT, and Kelsall RW. (2011) "Modulation of the absorption coefficient at $1.3 \mathrm{~mm}$ in Ge/SiGe multiple quantum well heterostructures on silicon," Opt. Lett. 36, 4158.

Livescu G, Miller DAB, Sizer T, Burrows DJ, Cunningham JE, Gossard AC, and English JH. (1989) "High-speed absorption recovery in quantum well diodes by diffusive electrical-conduction." Applied Physics Letters 54, 748.

Mahgerefteh D, Yang CM, Chen L, Hu KZ, Chen W, Garmire E, and Madhukar A. (1992) "Picosecond time-resolved measurements of electroabsorption in an InGaAs/GaAs multiple quantum-well p-i-n modulator." Applied Physics Letters 61, 2592.

McCallum DS, Cartwright AN, Huang XR, Boggess TF, Smirl AL, and Hasenberg TC. (1993) "Enhanced ambipolar inplane transport in an InAs/GaAs hetero-n-i-p-i." Journal of Applied Physics 73, 3860.

Miller DAB, Chemla DS, Damen TC, Gossard AC, Wiegmann W, Wood TH, and Burrus CA. (1985) “Electric-field dependence of optical-absorption near the band-gap of quantum-well structures." Physical Review B 32, 1043.

NASA Aeronautics Research Mission Directorate. (2014) “Achieving Our Vision: A New Approach for FY15," www.nasa.gov, NASA FY 2015 Budget Request Fact Sheets. 
Sato K, Abe T, Fujinuma R, Yasuda K, Yamaguchi T, Kasada H, and Ando K. (2012) "Stark effects of ZnO thin films and $\mathrm{ZnO} / \mathrm{ZnMgO}$ quantum wells," Phys. Status Solidi C 9, 1801.

Xu Z, Chen G, Abou-Galala F, and Leonardi M. (2007) "Experimental performance evaluation of non-line-of-sight ultraviolet communication systems," Proc. of SPIE 6709, 67090.

Yairi MB, Coldren CW, Miller DAB, and Harris JS. (1999) "High-speed, optically controlled surface-normal optical switch based on diffusive conduction." Applied Physics Letters 75, 597.

Zhang XY, Dhawan A, Wellenius P, Suresh A, and Muth JF. (2007) "Planar ZnO ultraviolet modulator," Appl. Phys. Lett. 91, 071107. 